\title{
Influence of a high electric field on the interaction of hydrogen with small palladium clusters: DFT studies
}

\author{
Agnieszka TOMASZEWSKA $^{1 *}$, Zdzisław STĘPIEN' ${ }^{1}$, Beata BIAŁEK ${ }^{1}$ \\ ${ }^{1}$ Institute of Physics, Jan Dtugosz University, Al. Armii Krajowej 13/15, 42-200 Czestochowa, Poland \\ * Corresponding author. Tel. +48-34-3614919; fax: +48-34-3668252; e-mail: a.tomaszewska@ajd.czest.pl
}

Received June 11, 2010; accepted May 18, 2011; available on-line November 8, 2011

The effect of an external electric field of $25 \mathrm{~V} / \mathrm{nm}$ on the interaction of atomic hydrogen with palladium clusters consisting of four and six atoms was investigated using the density-functional theory. The equilibrium geometry found for the systems under investigation is in good agreement with other computational studies carried out for extended systems, however the values obtained for the adsorption energy are overestimated. A comparison with the results obtained for clusters not affected by an external electric field shows that the effect of the field is a decrease of the energy barrier for incorporation of hydrogen into the clusters. The changes of the electronic structure induced by the electric field and the nature of the bonding between $\mathrm{H}$ and Pd atoms are analysed in a discussion of the density of states of the systems.

\section{Palladium / Hydrogen / DFT calculations}

\section{Introduction}

Palladium is one of the most often-used $d$-transition metals in the area of catalysis [1-2]. It supports organic synthesis [1], speeds up polymerization [3] and accelerates hydrogenation reactions because of its exceptional potential to absorb hydrogen. It is known that the presence of an external electric field considerably affects the metal surface reactivity [4-5]. In particular, a high electric field facilitates chemical reactions that do not easily occur under ordinary conditions. An example is the formation of $d$-transition metal hydrides [6-7]. In a low-temperature field evaporation experiment carried out in hydrogen atmosphere, Stępień and Tsong found that palladium hydrides were formed at the surface when an electric field of the magnitude of $26.7 \pm 0.8 \mathrm{~V} / \mathrm{nm}$ was applied [7].

Because of the importance of the interaction of hydrogen with $\mathrm{Pd}$ and the proved catalytic influence of an external electric field on the formation of metal hydrides, it would be of interest to find out how the electric field influences the nature of the interaction between hydrogen and Pd.

The interaction of atomic and molecular hydrogen with palladium surfaces not affected by an external field has been investigated in a wide variety of both experimental [8-12] and theoretical approaches [13-21]. In the experimental studies it was found that $\mathrm{H}$ atoms covering a palladium surface are rather weakly bound to the surface [12]. Computational investigations gave more detailed information on the mechanism and geometrical aspects of both adsorption of hydrogen on Pd surfaces and its diffusion into the $\mathrm{Pd}$ subsurface region $[15,17]$. In the computational studies of the interaction of hydrogen with Pd surfaces the substrate was modelled by either a supercell consisting of Pd layers separated by vacuum [17] or a two-dimensional slab [14], or a cluster [18-19].
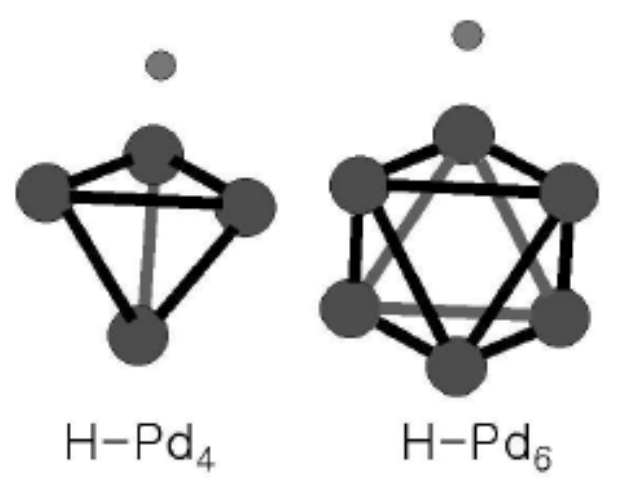

Fig. $1 \operatorname{Pd}_{n}(n=4,6)$ clusters. The small circles represent $\mathrm{H}$ atoms. 

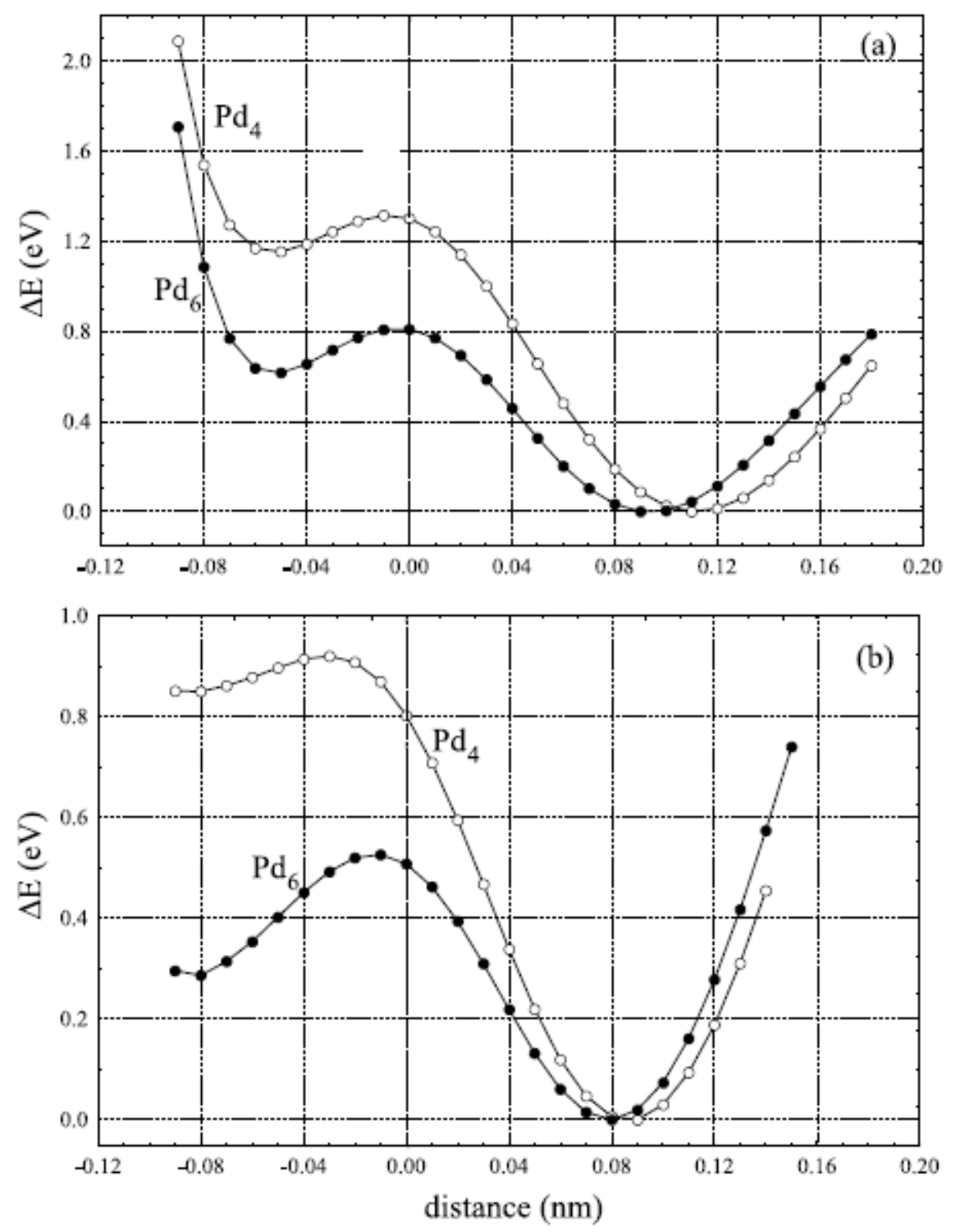

Fig. 2 Total energy change (with respect to the lowest energy of the system) as a function of the distance between the $\mathrm{H}$ atom and the $\mathrm{Pd}_{n}$ cluster surface: (a) no external field applied, (b) field intensity $25 \mathrm{~V} / \mathrm{nm}$. Zero distance corresponds to the cluster surface.

Roques et al. reported a comparison between some properties of small clusters and the (111) surface of palladium [20]. The authors applied the DFT method to study Pd clusters consisting of 3 to 13 atoms, bare or interacting with $\mathrm{H}$ and $\mathrm{O}$ atoms. From the results several parameters describing the properties of palladium were derived. The authors concluded that the chemisorption process of $\mathrm{H}$ on a $\mathrm{Pd}$ surface modelled by a cluster is satisfactorily described as far as the geometrical parameters are concerned, and in the case of three-dimensional clusters also the binding energy is in agreement with the values obtained in periodic-structure calculations. Although the values of binding energy were found to change slightly with the cluster size, 4- and 6-atom clusters appeared to be good models of the Pd(111) surface. In another work by Bertani et al. [18], devoted to investigations of 3to 7-atom Pd clusters interacting with hydrogen (and $\mathrm{CH}_{x}$ ), it was shown that adsorption of $\mathrm{H}$ atoms did not modify significantly the cluster geometry. The geometry of each studied cluster was optimized, and it was found that 4- and 6-atom clusters preferentially form a slightly distorted tetrahedron and octahedron, respectively. The distances between the $\mathrm{Pd}$ atoms in the clusters were close to those typical for such structures present at the $\operatorname{Pd}(111)$ surface, especially in the case of octahedral $\mathrm{Pd}_{6}$. It was also shown that the best agreement with experimental data was obtained 

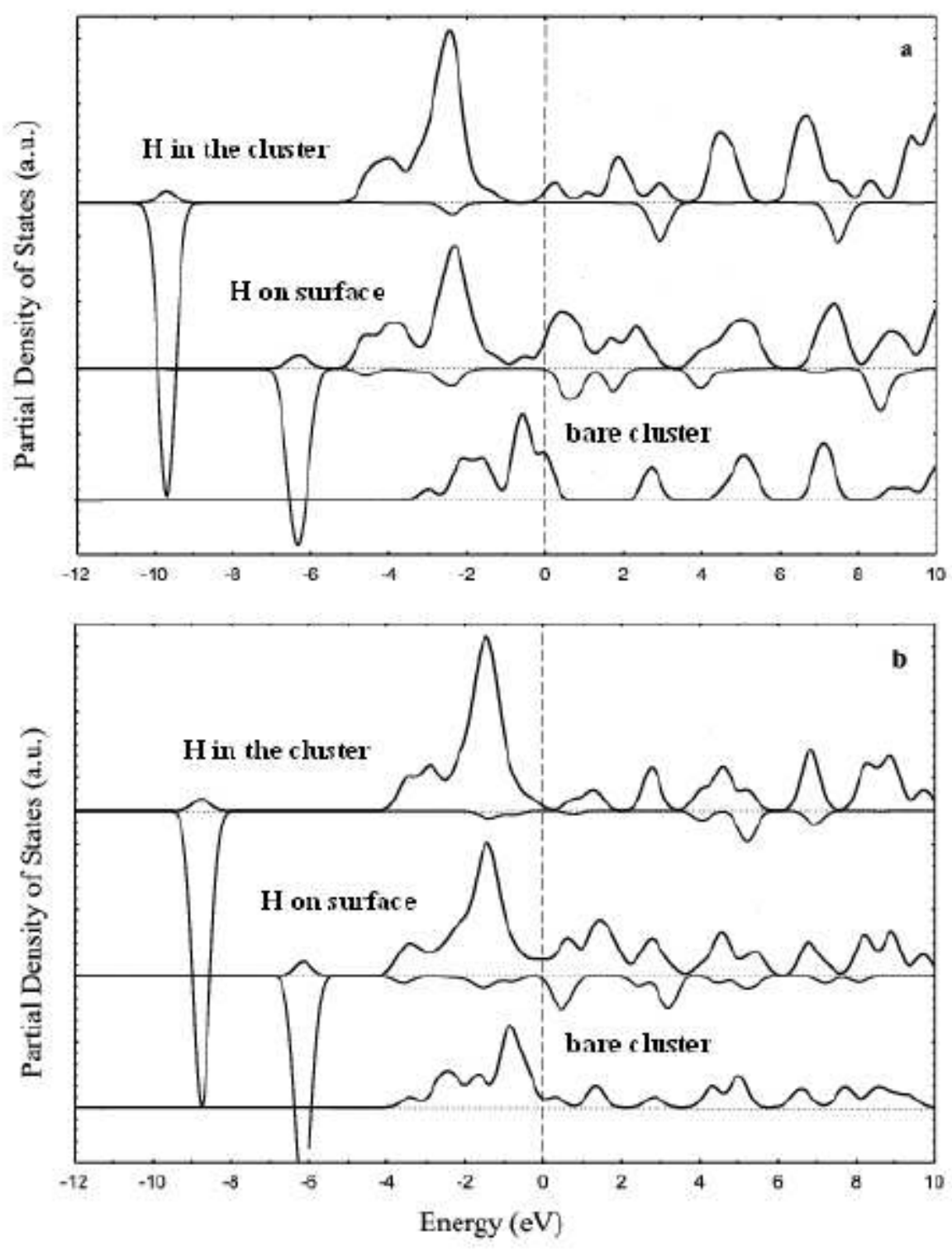

Fig. 3 Density of states distribution in a bare $\mathrm{Pd}_{4}$ cluster and $\mathrm{H}-\mathrm{Pd}_{4}$ complexes: (a) no external field, (b) field intensity $25 \mathrm{~V} / \mathrm{nm}$. Positive values show $\mathrm{Pd}_{4}$ pDOS, negative values $\mathrm{H}$ pDOS $(\times-30)$.

when a three-parameter Becke-Lee-Yang-Parr hybrid (B3LYP) exchange functional was used.

In this paper we present the results of a DFT study of the interaction of hydrogen with $\operatorname{Pd}_{n}(n=4$, 6) small clusters in the presence of a homogeneous electric field of the magnitude of $25 \mathrm{~V} / \mathrm{nm}$, which is comparable with that applied in the above mentioned experiment. Electric fields of this magnitude are experienced by valence electrons in atoms and molecules [22]. The effect of the electric field on the nature of the interaction between $\mathrm{H}$ and the two $\mathrm{Pd}$ clusters is analyzed in a discussion on the change of the total energy of the systems and the alteration of their electronic structure.

\section{Computational aspects}

We investigated the interaction of a single hydrogen atom with $\mathrm{Pd}$ clusters that correspond to two different sites of the (111) surface: the 4-atom cluster resembles a tetrahedral void (an hcp site) and the 6-atom one an octahedral void (an fcc site), as shown in Fig. 1. The distances between the cluster atoms were set to $0.275 \mathrm{~nm}$, which is close to the interatomic distances in the $\operatorname{Pd}(111)$ structure.

The presented results were obtained using the Gaussian 98 programme package [23]. We chose a density functional (DFT) approach with a hybrid B3LYP functional that includes a mixture of HartreeFock exchange with DFT exchange-correlation [24]. 

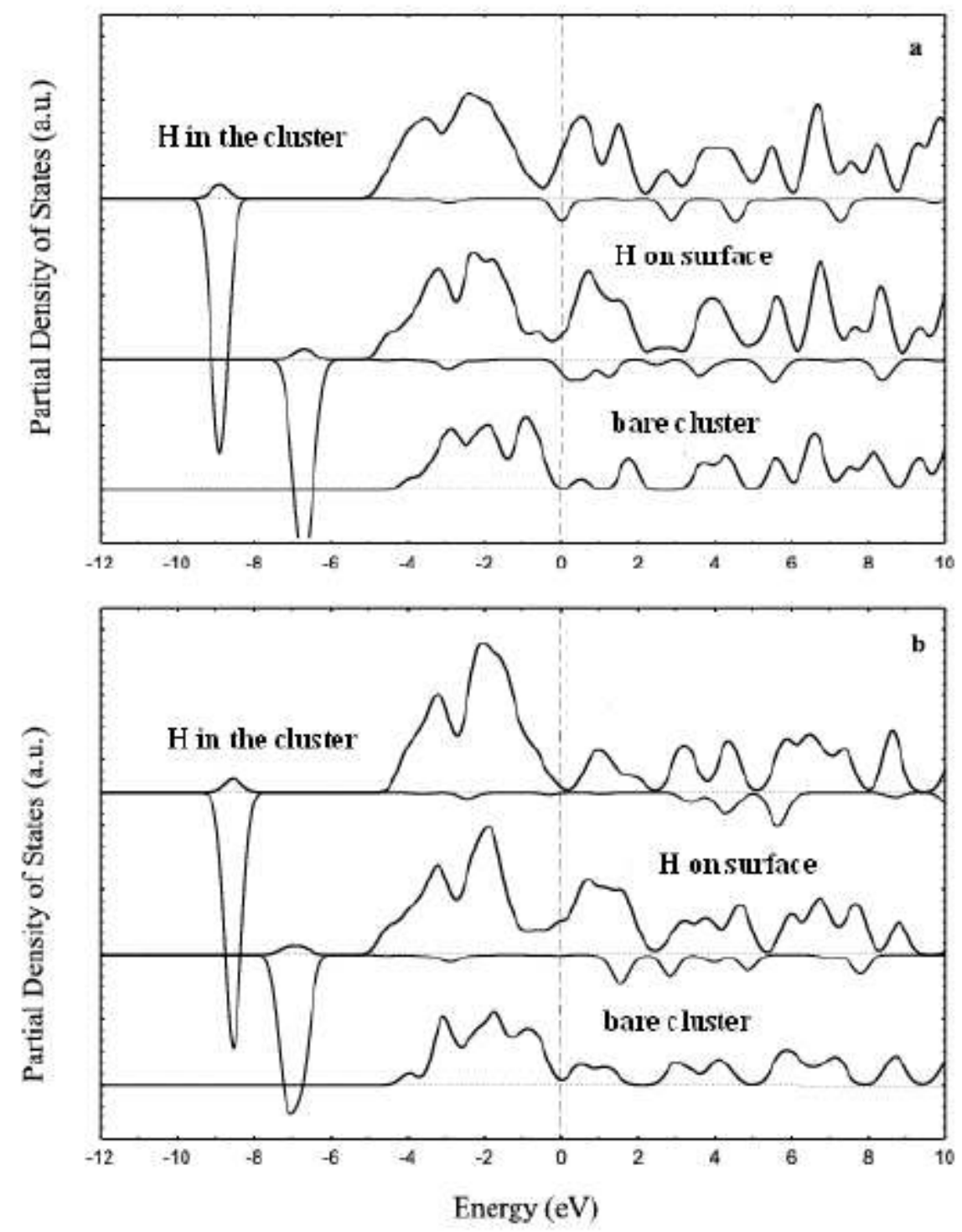

Fig. 4 Density of states distribution in a bare $\mathrm{Pd}_{6}$ cluster and $\mathrm{H}-\mathrm{Pd}_{6}$ complexes: (a) no external field, (b) field intensity $25 \mathrm{~V} / \mathrm{nm}$. Positive values show $\mathrm{Pd}_{6} \mathrm{pDOS}$, negative values $\mathrm{H}$ pDOS $(\times-30)$.

The use of this functional has already proved to give a reliable description of the interaction of hydrogen with small palladium clusters [18]. The Pd atoms were treated in the calculations in an approximate way, via electron core potentials, which include some relativistic effects important in heavy atoms. The chosen basis set was CEP-4G. Since we were first of all interested in the influence of a high electric field on the properties related to the adsorption of $\mathrm{H}$ on the $\mathrm{Pd}$ clusters, and taking into account the results mentioned in the introduction $[18,20]$, we did not perform any geometry optimization, neither of the bare clusters nor of those interacting with $\mathrm{H}$ atoms.

In order to consider the influence of a high electric field on the properties of the systems under study we added an external homogeneous electric field term in the electronic hamiltonian of the complexes. The electric field term was chosen in such a way that it described an electric dipole field in the direction perpendicular to the cluster surface towards the $\mathrm{H}$ atom. The density of the electronic states (DOS) was calculated for each of the investigated complexes in order to determine which changes in the electronic structure of the Pd clusters are caused by hydrogen adsorption and the presence of an electric field. The DOS, as well as spectra of crystal orbital overlap population (COOP) [25] that allow accounting for $\mathrm{H}-\mathrm{Pd}$ bonding, were obtained using the Gaussum 09 code [26].

\section{Results \\ Potential energy surface analysis}

On approaching a Pd cluster surface, the hydrogen atoms interact with the surface through either 
repulsive or attractive forces. The nature of the interaction may be examined with the use of quantumchemical calculations by an analysis of the dependence of the total energy $\left(E_{T}\right)$ on the distance between the $\mathrm{Pd}$ cluster and the $\mathrm{H}$ atom. Fig. 2 illustrates such dependencies obtained for the two investigated systems, i.e. $\mathrm{H}-\mathrm{Pd}_{4}$ and $\mathrm{H}-\mathrm{Pd}_{6}$, both in the case when no external electric field was present and when a field of $25 \mathrm{~V} / \mathrm{nm}$ was applied. Instead of $E_{T}$ values, the energy changes $(\Delta E)$ with respect to the minimum energy corresponding to the formation of a chemisorption bond between the $\mathrm{H}$ atom and the cluster are shown. The minimum energy was set to 0 .

The curves in Fig. 2 reveal the attractive nature of the interaction between the $\mathrm{H}$ atom and the $\mathrm{Pd}$ clusters. When no field is present $\Delta E$ shows a minimum when the $\mathrm{H}$ atom is situated $\sim 0.11(\sim 0.09)$ $\mathrm{nm}$ above the $\mathrm{Pd}_{4}\left(\mathrm{Pd}_{6}\right)$ cluster. These distances correspond to a bond length of $0.193(0.183) \mathrm{nm}$ between $\mathrm{H}$ and the nearest atoms, which is comparable with the values obtained from periodic-structure band structure calculations $(0.185$ and $0.181 \mathrm{~nm}$, respectively) [15].

When the $\mathrm{H}$ atom moves from its equilibrium position towards the inner part of a cluster, $E_{T}$ increases as long as the $\mathrm{H}$ atom is approaching the surface of the cluster. The value of $\Delta E$ corresponding to the maximum of the curve correlates with the activation energy for incorporating hydrogen inside the cluster. It is 1.30 and $0.82 \mathrm{eV}$ for $\mathrm{Pd}_{4}$ and $\mathrm{Pd}_{6}$, respectively. The lower activation energy for hydrogen penetration into the octahedral cluster is in agreement with the fact that $\mathrm{H}$ atom tends to occupy fcc subsurface sites more often than those of hcp symmetry [15].

When the $\mathrm{H}$ atom drifts into the cluster and moves towards its geometrical centre, $\Delta E$ decreases again reaching a local minimum at a distance close to the centre (Fig. 2a). The adsorption energy $\left(E_{a}\right)$ is calculated according to the formula:

$E_{a}=E_{P d_{n}}+E_{H}-E_{H-P d_{n}}$

where $E_{P d_{n}}, E_{H}$ and $E_{H-P d_{n}}$ are the total energies of the bare $\mathrm{Pd}_{n}$ cluster, hydrogen atom and $\mathrm{H}-\mathrm{Pd}_{n}$ system, respectively. The values -2.20 and $-2.25 \mathrm{eV}$ were obtained for $\mathrm{H}-\mathrm{Pd}_{4}$ and $\mathrm{H}-\mathrm{Pd}_{6}$, respectively. These values are smaller than $-2.69 \mathrm{eV}$, the experimental value obtained for $\mathrm{H}$ adsorption on a $\operatorname{Pd}(111)$ surface [8], and also different from the results obtained in other DFT studies, e.g. -2.48 for the hcp and $-2.65 \mathrm{eV}$ for the fcc site of $\operatorname{Pd}(111)$ [14]. This discrepancy indicates that it is necessary to check the effect of the basis set on the obtained results (for example, in a similar study of Pd clusters interacting with $\mathrm{H}$ atoms a better agreement with the experimental results was achieved with a more advanced basis set [20]), as well as the possible existence of lower energy states of other multiplicity than the singlet one considered in this work. Although the quantitative analysis of the obtained results is rather crude, we can still compare qualitatively the results obtained for the different clusters uninfluenced and influenced by an electric field. Fig. $2 b$ shows the influence of an external electric field on the energy of the investigated $\mathrm{H}-\mathrm{Pd}_{n}$ systems. In both cases the presence of the field shortens slightly (by $0.01 \mathrm{~nm}$ ) the equilibrium distance of the chemisorbed $\mathrm{H}$ atom to the cluster surface and the values of $E_{a}$ change to -1.53 and $-1.11 \mathrm{eV}$. This change in the strength of binding $\mathrm{H}$ to the clusters implies that a desorption process is more likely to occur in the presence of a high electric field. On the other hand, the activation barrier for penetration of $\mathrm{H}$ into the cluster decreases by 0.49 and $0.41 \mathrm{eV}$ for $\mathrm{Pd}_{4}$ and $\mathrm{Pd}_{6}$, respectively. Therefore, the migration of hydrogen into the clusters should be facilitated in the presence of the field. The local minima of $\Delta E$ on the energy curves corresponding to $\mathrm{H}$ positioned near the cluster centre are shallower than for the systems not influenced by the field. The clusters having incorporated an $\mathrm{H}$ atom appeared to be energetically unstable in the presence of the electric field when the $\mathrm{H}$ atom was moving from the point close to the geometrical centre of the cluster towards the cluster "subsurface" atoms. It is possible that in the presence of an external electric field the $\mathrm{H}$ atom is ionized and the proton moves freely inside the cluster. The charge distribution within the system should be taken into account in order to obtain a detailed description of the process.

\section{Density of states analysis}

To study the question of $\mathrm{H}$ bonding with $\mathrm{Pd}$ clusters we shall discuss the density of electronic states (DOS) of the investigated complexes and compare them with the DOS of non-hydrogenated clusters. The total DOS of the systems consisting of a $\mathrm{Pd}_{n}$ cluster and a single $\mathrm{H}$ atom is formed mainly by $\mathrm{Pd} 4 d$ states and the contribution of $\mathrm{H}$ is rather small. To make the picture of $\mathrm{H}$ partial DOS (pDOS) clearer, in Figs. 3 and 4 the states contributed by $\mathrm{H}$ are multiplied by -30 . The energy scale is chosen in such a way that 0 corresponds to the middle of the energy gap between the highest occupied and the lowest unoccupied molecular orbitals (HOMO and LUMO, respectively) of the investigated systems. This point on the energy scale is considered to correspond to the Fermi energy $\left(E_{F}\right)$ of the finite systems.

Fig. 3 shows the total DOS of a bare $\mathrm{Pd}_{4}$ cluster, as well as the partial $\mathrm{H}$ and $\mathrm{Pd}_{4}$ contributions to the DOS of an $\mathrm{H}-\mathrm{Pd}_{4}$ complex, not influenced by an electric field and with an applied electric field of $25 \mathrm{~V} / \mathrm{nm}$. There are several bands in the DOS spectrum of the bare $\mathrm{Pd}_{4}$ cluster. The occupied ones are formed by the valence electrons of Pd atoms localized in $4 d$ orbitals. They are seen in the energy range from -4 to $0 \mathrm{eV}$. The unoccupied states contribute to the bands at about 2.8, 5.0 and $7.0 \mathrm{eV}$.

The interaction of hydrogen with $\mathrm{Pd}_{4}$ clusters $(\mathrm{H}$ on surface) causes a rearrangement of the $\mathrm{Pd}_{4}$ energy 
levels. The gap between the HOMO and LUMO becomes larger, and the occupied states are moved deeper on the energy scale. The first band is localized around $-2.5 \mathrm{eV}$ and the number of states at $E_{F}$ is much lower than for the bare cluster. The observed reduction of the number of electronic states near the Fermi level is in agreement with earlier periodic-structure calculations and should be attributed to a removal of intrinsic surface states from this energy region $[13,17]$. This band, characterized by a considerable number of states contributed by the $\mathrm{H}$ atom, is seen at about $-6.2 \mathrm{eV}$. At the same energy there are other electron states contributed by the $\mathrm{Pd}_{4}$ cluster. The strong hybridization of the orbitals of $\mathrm{Pd}_{4}$ and $\mathrm{H}$ is the result of the interaction between $\mathrm{H} 1 s$ and $d$ states of $\mathrm{Pd}_{4}$. The interaction, resulting in the presence of electron states below $-6.0 \mathrm{eV}$, is of bonding character, which was confirmed by an orbital overlap population analysis. The presence of a new peak below the bottom of the $d$ band at about $-6.2 \mathrm{eV}$ corresponds to the new surface induced by $\mathrm{H}$ adsorption [14].

Hydrogen chemisorption on the $\mathrm{Pd}_{4}$ cluster surface changes considerably the picture of the unoccupied electron states. There are more bands in the considered energy range (above the 0 level) coming from the interactions between empty $\mathrm{H}$ states and $\mathrm{Pd}_{4}$ molecular orbitals. The abundance of new empty states near the 0 level is consistent with the higher catalytic activity of hydrogenated palladium.

When hydrogen overcomes the potential barrier and penetrates into the $\mathrm{Pd}_{4}$ bulk, the shape of the overall DOS changes again. The band with contribution from $\mathrm{Pd} 4 d$ states becomes narrower, and the number of states at $-2.8 \mathrm{eV}$ is larger, which means that the electronic levels lay closer to each other; due to the presence of hydrogen inside the void, their number is larger. The band that represents the bonding interactions between the $\mathrm{H}$ atom and the atoms forming the $\mathrm{Pd}_{4}$ cluster is moved $3.6 \mathrm{eV}$ deeper on the energy scale with respect to the corresponding band for the $\mathrm{Pd}_{4}$ cluster adsorbing an $\mathrm{H}$ atom. It is narrower than in the case of $\mathrm{Pd}_{4}$ chemisorbing $\mathrm{H}$, i.e. even more localized. The number of $\mathrm{H}$ states in the band is almost twice larger, which is probably due to the interaction of the $\mathrm{H}$ atom with not only the surface of the cluster, but also the fourth $\mathrm{Pd}$ atom. The unoccupied part of the DOS spectrum becomes similar to the DOS of the bare cluster. The bands are clearly distinguished, especially those at 4.8 and $6.8 \mathrm{eV}$ above the 0 level. The number of states in the bands close to the 0 level is much smaller than that observed for the cluster with chemisorbed $\mathrm{H}$, which is probably due to the screening effect of $\mathrm{Pd}$ atoms surrounding the hcp site occupied by an $\mathrm{H}$ atom.

Fig. 3b illustrates how the applied electric field modifies the electronic structure of the $\mathrm{Pd}_{4}$ and $\mathrm{H}-\mathrm{Pd}_{4}$ systems. The shape of the DOS of the bare cluster in the energy range from -4.0 to $-1.0 \mathrm{eV}$ is slightly changed due to splitting of the $t_{2 g}$ levels of the cluster in the presence of the field. Besides, the unoccupied part of the DOS curve differs considerably from that of the cluster without applied field. There are more features in the spectrum, but their intensity is lower.

When the electric field is applied, the presence of $\mathrm{H}$ in the vicinity of $\mathrm{Pd}_{4}$ causes effects similar to those described above for the cluster without applied field, especially for to the occupied energy levels. Due to the interaction with the $\mathrm{H}$ atom, the band formed mainly by $d$ electrons of $\mathrm{Pd}_{4}$ is wider than that of the bare cluster, and its intensity is larger. It is noticeable that the relative positions of the wide $d$-band of $\mathrm{Pd}_{4}$ and the split-off band are changed due to the presence of the field, which may correspond to changes of the band structure of the system. The unoccupied part of the DOS curve is characterized by several features, more than for the cluster not affected by the field. The splitting of the bands is accompanied by lower intensity. The presence of the electric field moves the second band contributed by unoccupied states of $\mathrm{H}$ by more than $1.0 \mathrm{eV}$ along the energy scale, and other unoccupied states almost vanish. The weaker hybridization observed between $\mathrm{H}$ and $\mathrm{Pd}_{4}$ orbitals implies that the chemisorption bond between $\mathrm{H}$ and the cluster is weakened as well, and the $\mathrm{H}$ atom can easier migrate into the void. Once the atom is inside the void, the character of the interaction with the cluster atoms is similar to what was observed with no field present. The localized band contributed by $\mathrm{H} 1 \mathrm{~s}$ and $\mathrm{Pd}_{4} 4 d$ states is moved by $\sim 1 \mathrm{eV}$ closer to $E_{F}$. A weak hybridization between empty states of $\mathrm{H}$ and $\mathrm{Pd}_{4}$ is noticed only at $5.2 \mathrm{eV}$.

As it is seen from Fig. 4, the changes in the DOS curve due to the interaction of $\mathrm{H}$ with the surface of the $\mathrm{Pd}_{6}$ cluster are similar to those discussed in the case of the $\mathrm{H}-\mathrm{Pd}_{4}$ system. The structure of the empty electronic states is changed to quite a large extent: a wide unoccupied band with a large number of states appears close to the zero energy level. The positions of the other unoccupied bands correspond well to those of the bare $\mathrm{Pd}_{6}$ cluster.

Unlike $\mathrm{H}-\mathrm{Pd}_{4}$, when $\mathrm{H}$ immigrates into the octahedral void the pDOS picture of $\mathrm{Pd}_{6}$ remains almost unchanged in the whole energy range, as compared with $\mathrm{Pd}_{6}$ chemisorbing $\mathrm{H}$. $\mathrm{H}$ pDOS changes considerably in its unoccupied part in such a way that there are less bands of more localized character (at about $0,3.0,4.5$, and $7.5 \mathrm{eV}$ ).

The presence of an external high electric field does not influence strongly the total DOS of the bare $\mathrm{Pd}_{6}$ cluster (Fig. 4b), but it changes the electronic structure of the cluster interacting with an $\mathrm{H}$ atom. The presence of the field reduces the number of $\mathrm{H}$ states at $-7.0 \mathrm{eV}$ by $\sim 20 \%$ and the band is wider. However, the relative positions of the split-off band and the $d$-band of the cluster are not changed as much as for the $\mathrm{H}-\mathrm{Pd}_{4}$ system. In the unoccupied part of the spectrum a reduction of the band separation is seen in the pDOS formed from unoccupied states of the cluster. When $\mathrm{H}$ migrates into the octahedral void in the presence of the electric field most of the $\mathrm{H}$ states 
are suppressed, and so are the states originating from the $\mathrm{Pd}_{6}$ cluster.

\section{Conclusions}

The influence of a high electric field on the interaction of atomic hydrogen with small Pd clusters was investigated using the DFT method. The field magnitude was $25 \mathrm{~V} / \mathrm{nm}$. Although small clusters are not representative of a $\mathrm{Pd}$ surface, the geometrical parameters obtained in our calculations are in good agreement with both experimental and theoretical data. The values obtained for the adsorption energy differ, however, from those available in the literature. Nevertheless, an analysis of the relative change of the total energy of the systems indicates that the energy barrier for hydrogen incorporation into the cluster decreases in the presence of an electric field. The curves of the density of states obtained for the investigated clusters without applied field (both bare and interacting with $\mathrm{H}$ ) confirms that $\mathrm{H}$ adsorption causes a considerable reduction of the number of states near the Fermi level. The presence of a field changes the density of unoccupied states to a larger extent than the density of occupied states. The results indicate that an external electric field may promote catalytic reactions, especially those that involve interaction with hydrogen.

\section{Acknowledgement}

The authors are indebted to Prof. J.I. Lee for giving BB an opportunity to work in his laboratory (Inha Univ., Incheon, Korea).

\section{References}

[1] J. Tsuji, Palladium Reagents and Catalyst, Wiley, Chichester, UK, 1996.

[2] H.U. Blaser, A. Indolese, A. Schnyder, H. Steiner, M. Studer, J. Mol. Catal. A 173 (2001) 3 .

[3] G. Müller, B. Rieger, Prog. Polym. Sci. 27 (2002) 815.

[4] T.T. Tsong, Field Ion Microscopy, Cambridge University Press, 1990.

[5] J.H. Block, H.J. Krauzer, L.C. Wang, Surf. Sci. 246 (1991) 125.

[6] T.T. Tsong, T.J. Kinkus, Phys. Rev. 29 (1984) 529.
[7] Z.M. Stępień, T.T. Tsong, Surf. Sci. 409 (1998) 57.

[8] H. Conrad, G. Ertl, E.E. Latta, Surf. Sci. 41 (1974) 435.

[9] K.R. Christmann, Surf. Sci. Rep. 9 (1988) 1.

[10] T.E. Felter, E.C. Sowa, M.A. van Hove, Phys. Rev. B 40 (1989) 891.

[11] T. Mitsui, M.K. Rose, E. Fomin, D.F. Ogletree, M. Salmeron, Surf. Sci. 511(2002) 259.

[12] S. Horinouchi, Y. Yamanoi, T. Yonezawa, T. Mouri, H. Nishihara, Langmuir 22 (2006) 1880.

[13] S.G. Louie, Phys. Rev. Lett. 42 (1979) 476.

[14] J.F. Paul, P. Sautet, Phys. Rev. B 53 (1996) 8015.

[15] O.M. Løvvik, R.A. Olsen, Phys. Rev. B 58 (1998) 10890.

[16] W. Dong, J. Hafner, Phys. Rev. B 56 (1997) 15396.

[17] W. Dong, V. Ledentu, Ph. Sautet, A. Eichler, J. Hafner, Surf. Sci. 411 (1998) 123.

[18] V. Bertani, C. Cavallotti, M. Masi, S. Carra, J. Phys. Chem. A 104 (2000) 11390.

[19] I. Efremenko, J. Mol. Catal. A 173 (2001) 19.

[20] J. Roques, C. Lacaze-Dufaure, C. Mijoule, Surf. Sci. 479 (2001) 231.

[21] M. Lischka, A. Groß, Phys. Rev. B 65 (2002) 075420 .

[22] H.J. Kreuzer, Surf. Sci. 246 (1991) 336.

[23] M.J. Frisch, G.W. Trucks, H.B. Schlegel, G.E. Scuseria, M.A. Robb, J.R. Cheeseman, V.G. Zakrzewski, J.A. Montgomery, Jr., R.E. Stratmann, J.C. Burant, S. Dapprich, J.M. Millam, A.D. Daniels, K.N. Kudin, M.C. Strain, O. Farkas, J. Tomasi, V. Barone, M. Cossi, R. Cammi, B. Mennucci, C. Pomelli, C. Adamo, S. Clifford, J. Ochterski, G.A. Petersson, P.Y. Ayala, Q. Cui, K. Morokuma, D.K. Malick, A.D. Rabuck, K. Raghavachari, J.B. Foresman, J. Cioslowski, J.V. Ortiz, B.B. Stefanov, G. Liu, A. Liashenko, P. Piskorz, I. Komaromi, R. Gomperts, R.L. Martin, D.J. Fox, T. Keith, M.A. Al-Laham, C.Y. Peng, A. Nanayakkara, C. Gonzalez, M. Challacombe, P.M.W. Gill, B. Johnson, W. Chen, M.W. Wong, J.L. Andres, C. Gonzalez, M. Head-Gordon, E.S. Replogle, J.A. Pople, Gaussian 98, Revision A.6. Gaussian Inc., Pittsburg, PA, 1998.

[24] A.D. Becke, J. Chem. Phys. 98 (1993) 5648.

[25] R. Hoffmann, Solids and Surfaces: A Chemist's View of Bonding in Extended Structures, VCH Publishers, NY, 1988.

[26] N. O’Boyle, http://gaussum.sourceforge.net.

Proceeding of the XVI International Seminar on Physics and Chemistry of Solids,

Lviv, June 6-9, 2010. 